LETTER TO EDITOR

\title{
Intravenous Fosfomycin for Treatment of Refractory Stenotrophomonas maltophilia Ventriculitis
}

\author{
Veronica Salazar ${ }^{1}$, Laura Aragon ${ }^{2}$, Lilian Abbo ${ }^{3}$ \\ ${ }^{1}$ Department of Pharmacy, Jackson Memorial Hospital, Miami, FL, USA \\ ${ }^{2}$ Department of Pharmacy, Saint Luke's Health System, Kansas City, MO; USA \\ ${ }^{3}$ University of Miami Miller School of Medicine, Miami, FL, USA
}

J Microbiol Infect Dis 2020; 10(2): 106-107.

Keywords: Fosfomycin, Combination, Stenotrophomonas maltophilia, Ventriculitis

\section{Dear Editor,}

We read a review article published by Karadağ et al [1] on the use of Fosfomycin as an alternative to treat gram-positive and gramnegative bacteria, including multidrug resistant isolates. Intravenous (IV) fosfomycin has been shown to be a safe and effective alternative in treating infections outside of the urinary and gastrointestinal tract [2]. As discussed in Kastoris et al [3], the synergistic combination of IV fosfomycin with other antibiotics is a promising alternative in many difficult infections.

In the United States, about 2 million people are infected with antibiotic-resistant bacteria and at least 23,000 people die as a result [4]. Multidrug resistant (MDR) pathogens are becoming a challenge to treat due to the limited number of antibiotics with novel mechanisms of action against Pseudomonas and non-enteric gramnegative organisms being introduced into the market; and the rapid development of resistance to the new and old drugs. As a result, combination therapy has become a necessity to treat many of these MDR infections usually guided by microbiological synergy or empiric clinical desperation, especially in critically ill patients. In the United States, fosfomycin is only commercially available orally as to treat uncomplicated urinary tract infections [6]. IV Fosfomycin is available in many countries outside of the United States, however in order to use the IV formulation an investigational new drug application (IND) is required and must be approved by the Food and Drugs Administration (FDA). We present a case where microbiological cure was achieved in a 33 year-old male admitted due to a facial gunshot wound status post decompressive craniotomy whose hospital course was complicated secondary to refractory Stenotrophomonas maltophilia ventriculitis. During hospitalization patient was noted to be colonized with MDR Pseudomonas aeruginosa, S. maltophilia, and Acinetobacter baumanii and had been exposed to several courses of antibiotics including vancomycin, meropenem, levofloxacin, and ceftazidime. On day 32, patient's mental status worsened, cerebrospinal fluid (CSF) culture was done and he was started on IV Ceftazidime $2 \mathrm{~g}$ IV every 8 hours and intrathecal (IT) colistin pending antibiotic susceptibility results. CSF culture was positive for S. maltophilia resistant to sulfamethoxazole/trimethoprim (SMX-TMP) and levofloxacin. Further susceptibility testing done and the following were reported: minocycline (minimum inhibitory concentration $[\mathrm{MIC}]=4$ $\mu \mathrm{g} / \mathrm{mL})$, ceftazidime $(\mathrm{MIC}=8 \mu \mathrm{g} / \mathrm{mL})$, tigecycline $(\mathrm{MIC}=8 \mu \mathrm{g} / \mathrm{mL})$, fosfomycin $(\mathrm{MIC}=32 \mu \mathrm{g} / \mathrm{mL})$, and colistin ( $\mathrm{MIC}=256 \mu \mathrm{g} / \mathrm{mL}$ ) for which there are currently no MIC breakpoints for $S$. maltophilia for tigecycline, fosfomycin or colistin per CLSI. At this point, based on susceptibilities, colistin was discontinued and minocycline IV $100 \mathrm{mg}$ every 12 hours and fosfomycin $3 \mathrm{~g}$ oral every 24 hours was added to ceftazidime. On 
day 43 , while on current antibiotic regimen, patient became febrile and CSF cultures were repeated and were positive once again for $S$. maltophilia. Due to limited treatment options, synergy test for IV fosfomycin and ceftazidime was requested. The fosfomycin dose was increased to $3 \mathrm{~g}$ orally every 8 hours and IV rifampin $600 \mathrm{mg}$ IV daily was added to the regimen pending FDA approval for IV fosfomycin. On day 49, IV fosfomycin arrived from Nordic Pharma in Europe and oral formulation was switched to IV $4 \mathrm{~g}$ every 6 hours. After three days of IV fosfomycin in combination with IV minocycline and IV rifampin, cultures became negative. After several months in the hospital and no meaningful recovery, the patient was transitioned to hospice for comfort measures.

IV fosfomycin has been a successful alternative in the treatment of many gram-positive and gram-negative infections in several articles that have been published outside the US and has excellent penetration in the CSF in which increased concentrations can be achieved in meningeal inflammation [2,5]. In our case, testing in vitro the activity of combination therapy is also important, and we recommend in vitro testing to ensure that there is no antagonism and that there will be a high likelihood of clinical success and not driven by empiric choices. Unfortunately, obtaining IV fosfomycin from Europe is a difficult process that could be detrimental to timely patient care when treating difficult infections. As the emergence of antimicrobial resistance continues to rise, we need new antibiotics and stewardship of antimicrobial testing to define the ideal combinations of therapy when treating patients with challenging infections.

Correspondence: Dr. Veronica Salazar, Jackson Memorial Hospital, Department of Pharmacy, Miami, FL, USA

Email: Veronica.salazar@jhsmiami.org

\section{ACKNOWLEDGMENTS}

Declaration of Conflicting Interests: The authors declare that they have no conflict of interest.

Financial Disclosure: No financial support was received.

\section{REFERENCES}

1. Karadağ A, Çaycı $Y T$, Bilgin $K$, Günaydın $M$, Eroğlu C. In vitro efficacy of fosfomycin against clinical strains. J Microbiol Infect Dis 2014; 4(2):55-58.

2. Falagas ME, Giannopoulou KP, Kokolakis GN, Rafailidis PI. Fosfomycin. Use Beyond Urinary Tract and Gastrointestinal Infections. Clin Infect Dis 2008; 46(7):1069-1077.

3. Kastoris AC, Rafailidis PI, Vouloumanou EK, Gkegkes ID, Falagas ME. Synergy of fosfomycin with other antibiotics for Gram-positive and Gramnegative bacteria. Europ J Clin Pharma 2010; 6 6(4):359-368.

4. Antibiotic Resistance Threats - cdc.gov. https://www.cdc.gov/drugresistance/pdf/ar-threats2013-508.pdf. Accessed August 23, 2019.

5. Pfausler $B$, Spiss $H$, Dittrich $P$, Zeitlinger $M$, Schmutzhard E, Joukhadar C. Concentrations of fosfomycin in the cerebrospinal fluid of neurointensive care patients with ventriculostomyassociated ventriculitis. J Antimicrob Chemother. 2004; 53(5):848-852. 